\title{
FIBROSIS RETROPERITONEAL POR PANCREATITIS EN PACIENTE VIH+
}

\author{
M.A. GARCÍA GARCÍA, M.A. TRÍVEZ BONED, C. SANCHO, E. MALlÉN MATEO, \\ C. RIOJA SANZ, L.A. RIOJA SANZ
}

Servicio de Urología. Hospital Universitario Miguel Servet. Zaragoza.

Actas Urol Esp. 27 (6): 458-461, 2003

\section{RESUMEN}

FIBROSIS RETROPERITONEAL POR PANCREATITIS EN PACIENTE VIH+

La fibrosis retroperitoneal es un raro proceso inflamatorio crónico, que provoca la compresión de estructuras debido al desarrollo de una placa fibrótica.

Existen diferentes etiologías, entre las que destacan las enfermedades inflamatorias, tanto específicas como inespecíficas. Dentro de las primeras se situaría la producida por la pancreatitis.

Presentamos un raro caso de fibrosis retroperitoneal debido a una pancreatitis en un paciente $\mathrm{VIH}+$.

PALABRAS CLAVE: Fibrosis retroperitoneal. Pancreatitis. VIH.

\section{ABSTRACT}

RETROPERITONEAL FIBROSIS DUE TO PANCREATITIS IN HIV+ PATTENT

Retroperitoneal fibrosis is a rare inflammatory disease, in which the fibrosis plate leads to the compression of the affected structures.

There are several causes of retroperitoneal fibrosis like specific and unspecific inflammatory diseases. In the specific group of retroperitoneal fibrosis we find the one due to pancreatitis.

We do present a rare case of retroperitoneal fibrosis caused by a pancreatitis in a HIV+ patient. KEY WORDS: Retroperitoneal fibrosis. Pancreatitis. VIH.

$\mathrm{L}^{\mathrm{a}}$ a fibrosis retroperitoneal es un proceso no muy frecuente dentro de la práctica clínica habitual. Existen diferentes etiologías responsables, entre las que caben destacar las de enfermedades colindantes inflamatorias, dentro de las cuales se encuentra la pancreatitis.

Si bien no es una patología fácil de encontrar, menos aún en el contexto de un paciente VIH+, que presenta una pancreatitis crónica reagudizada, como es el caso que nos ocupa.

\section{HISTORIA CLÍNICA}

Paciente de 30 años con trastorno esquizoafectivo. ADVP. VIH+, diagnosticado desde el año 1997 en tratamiento por parte del Servicio de Medicina Interna. Dolores difusos abdominales de un año de evolución.
Pancreatitis aguda hace 3 años evolucionando a cronicidad.

No presenta ningún antecedente urológico de interés.

Ingresado en el Servicio de Infecciosos ante cuadro de astenia, anorexia, adelgazamiento y síndrome febril de $38^{\circ}$. Amilasas en sangre y en orina elevadas.

Es diagnosticado de reagudización de pancreatitis crónica con una evolución tórpida.

Las analíticas denotan un componente inflamatorio, presentando una VSG de 120.

En una ecografía se aprecia un sistema excretor derecho sin anomalias. En el sistema excretor izquierdo se aprecia una hidronefrosis marcada grado III-IV, razón por la que nos es requerida nuestra colaboración. 
En UIV se aprecia gran dilatación aguda en pelvis y grupos calicilares con estrechez patente a nivel de la unión pielo-ureteral. No se aprecian defectos de repleción ni en pelvis ni en el trayecto ureteral.

Se decide ante tales hallazgos la derivación del sistema excretor izquierdo mediante la realización de nefrostomía percutánea izquierda, con salida de orina a ritmo retentivo.

En TAC en la fase parenquimatosa (Fig.1), se aprecia páncreas de morfología conservada, discretamente aumentado de tamaño. Signos de edema de las estructuras peri pancreática, incluyendo fascia renal izquierda y mesenterio con signos de pseudoencapsulación y pequeños nódulos mesentéricos. Colon transverso de pared engrosada con signos de espasticidad e hipervascularización y edema perivisceral.

El sistema excretor derecho no presenta alteraciones. En el sistema excretor izquierdo, se aprecia una dilatación grado III-IV. En la fase excretora (Fig. 2) engrosamiento parietal de la pelvis renal dilatada. Edema de la grasa retroperitoneal pararrenal anterior y posterior junto a las estructuras peripancreáticas. En cortes más bajos englobamiento del trayecto ureteral izquierdo.

En pielografía descendiente se confirma la dilatación pielo-calicilar, con estenosis a nivel de la unión pielo-ureteral, obstruyendo el paso del contraste.

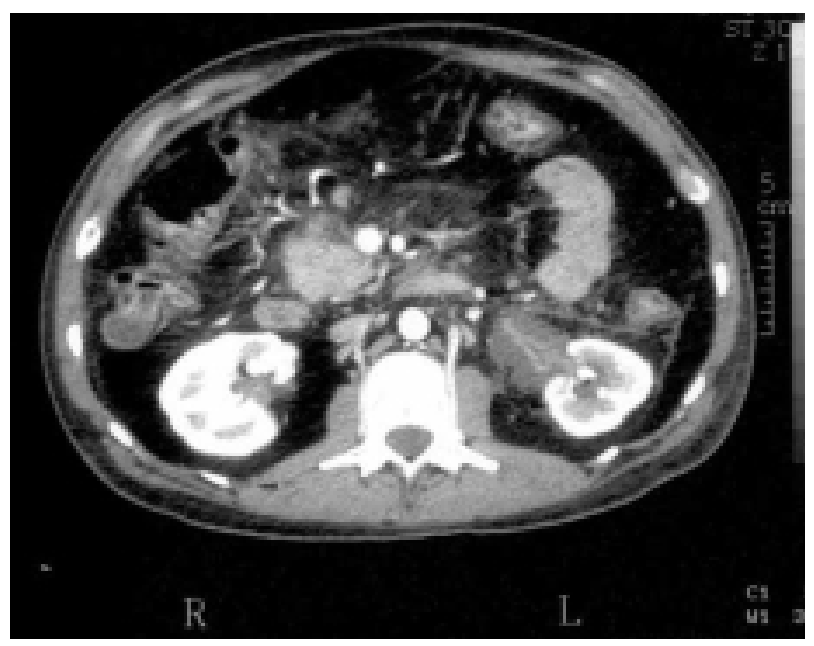

FIGURA 1. T.A.C. Se aprecian en fase parenquimatosa signos de edema de las estructuras peripancreáticas y mesentéricas junto con edema de la grasa retroperitoneal. Pelvis renal dilatada y extrasinusal.

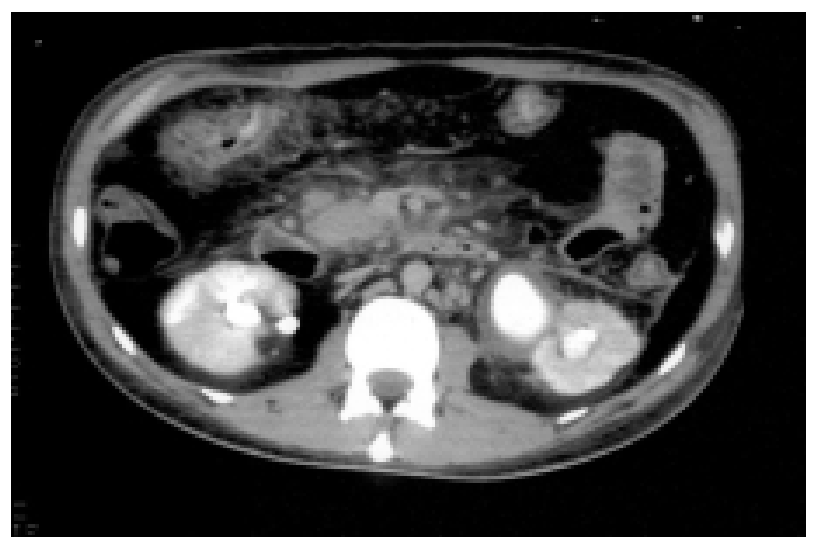

FIGURA 2. T.A.C en fase de eliminación. Signos edematosos peripancreáticos y retroperitoneales (inclusive cólon transverso). Dilatación de pelvis renal, con engrosamiento parietal de la misma.

Ante todos éstos hallazgos se deciden exploración ureteral, siendo necesaria la dilatación del meato ureteral mediante catéter-balón debido a la existencia de una estenosis moderada, debido a la compresión inflamatoria extraureteral. A nivel de la unión se aprecia una severa estenosis que no permite el paso del ureterorrenoscopio, siendo posible tan solo el paso de guía Terumo ${ }^{\circledR}$, a través de la cual se consigue la colocación de un catéter pig-tail ${ }^{\circledR}$. No se apreciaron litiasis a lo largo de todo el trayecto excretor (Fig. 3).

Se instaura tratamiento corticoide con metilprednisolona (Urbasón ${ }^{\circledR}$ ) $40 \mathrm{mg} /$ día durante el primer mes, con la intención de ir descendiendo en el segundo mes a una dosis de $20 \mathrm{mg}$ /día y de pautar con posterioridad una dosis de mantenimiento de $8 \mathrm{mg} /$ día durante los meses siguientes. Asimismo se pretende el mantenimiento del catéter pig-tail hasta ver una correcta evolución del proceso y una respuesta adecuada al tratamiento corticoide.

La evolución posterior del paciente en las semanas siguientes es infausta a consecuencia de fallo multiorgánico debido a la mala evolución del proceso de base, conduciendo al exitus del paciente.

\section{DISCUSIÓN}

La fibrosis retroperitoneal es un proceso poco frecuente, caracterizado por presentar a nivel retroperitoneal prevertebral un componente de origen inflamatorio con una evolución fibrótica. 

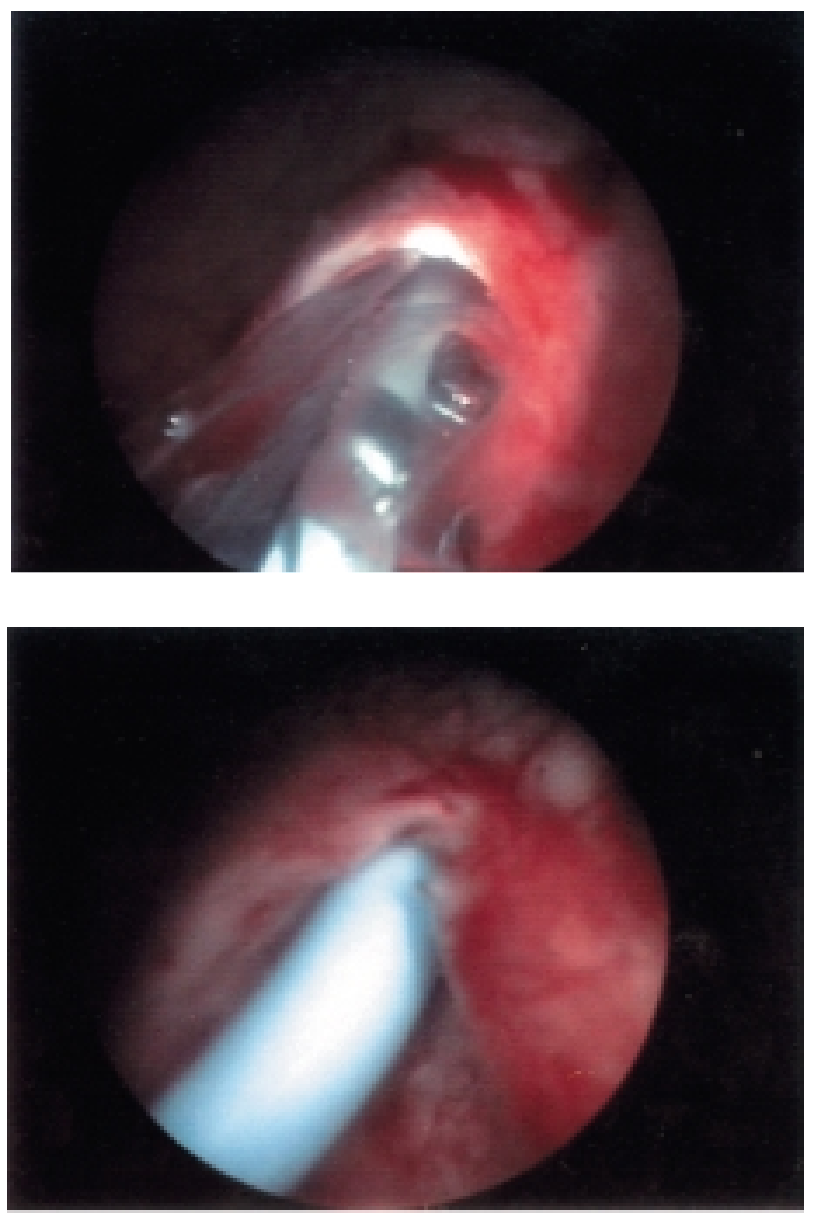

FIGURA 3. Dilación del meato ureteral y posterior paso de catéter pig-tail.

Como consecuencia de dicho proceso se produce el atrapamiento de uréteres, linfáticos, venas y arterias a los que comprime ${ }^{1}$.

Presenta una clarísima preferencia por el sexo masculino, siendo aproximadamente de 3 a 1 la relación existente.

No se conocen bien los mecanismos etiopatogénicos que la producen. En 1972 Mitchinson describe que pudiera deberse a una reacción autoinmune frente a un antígeno situado en las placas ateromatosas de la aorta abdominal ${ }^{2,3}$.

Existen hoy en día descritas diversas etiologías responsables de éste proceso:

I. Idiopática. $43 \%$

II. Drogas. 3\% (LSD, metisergida, reserpina, metildopa, fenacetina, anfetaminas, etc.)

III. Procesos malignos. $12 \%$ (primarios ó secundarios)

IV. Procesos inflamatorios. 7\% a) no específicos (enfermedad del colágeno vascular autoinmune, periarteritis, urinomas, aneurisma inflamatorio aórtico, etc.)

b) específicas (sarcoidosis, endometriosis, inflamaciones gastro-inflamatorias, pancreatitis, etc.)

V. Hemorrágica. 15\% (postraumática, posquirúrgico)

VI. Radioterapia. $2 \%$

VII. Miscelánea. 18\% (infecciosa, etc.)

Dentro de los procesos inflamatorios específicos se encontraría la pancreatitis como causante de ésta patología. Lewis el al. describen la relación de la pancreatitis con la trombosis secundaria de la vena esplénica y con la fibrosis retroperitoneal ${ }^{4}$.

Reynaud el al. presentan el caso de un paciente con pancreatitis, el cual desarrolla una fibrosis retroperitoneal con estenosis ureteral de predominio izquierdo ${ }^{5}$. En una revisión comentada por el autor, la incidencia de casos es muy pequeña en la literatura, no encontrándose ningún paciente $\mathrm{VIH}+$. Según Raynaud la fibrosis retroperitoneal inducida por ésta patología se debería a dos mecanismos etio-patogénicos fundamentalmente. Un primer mecanismo que sería la compresión mecánica por parte del páncreas y un segundo mecanismo que explicaría la patología mediante una necrosis isquémica ó enzimática que conllevaría posteriormente a una fibrosis secundaria.

Ruckert el al. hacen también posteriormente una descripción de un par de casos acaecidos en su Servicio ${ }^{6}$, comentando la poca frecuencia de ésta patología.

Las manifestaciones clínicas sistémicas que se ponen de manifiesto en ésta patología como son el dolor abdominal difuso, la anorexia, la pérdida de peso y la fiebre, están en éste caso que nos ocupa enmascaradas por la similar clínica del proceso de base.

El dolor es el síntoma inicial más frecuente referido por los enfermos ${ }^{7}$. Puede ser lumbar en un $40-60 \%$ ó abdominal difuso en un 50\%.

En general las alteraciones analíticas son inespecíficas, siendo la elevación de la velocidad de sedimentación globular por encima de 30 $\mathrm{mm} / \mathrm{h}$ la única constante a tener en cuenta en estos pacientes ${ }^{2}$. 
El T.A.C. junto con la U.I.V, continúan siendo las dos exploraciones fundamentales en el diagnóstico y seguimiento de ésta patología.

El tratamiento debe estar orientado tanto a paliar el proceso etiológico (en nuestro caso se trataría de la pancreatitis crónica de base con sus episodios de reagudización), como a tratar el proceso inflamatorio y el consiguiente proceso obstructivo renal desencadenado.

La colocación de un catéter pig-tail o de una nefrostomía de descarga, es una medida que nos asegura un correcto funcionalismo renal, evitando además la obstrucción progresiva durante la evolución del proceso. El tiempo que se debe mantener está en relación con la evolución del proceso y el resultado del tratamiento ${ }^{2}$.

El mejor valor analítico para el seguimiento y la evolución de la enfermedad son las cifras de VSG.

El tratamiento médico con metilprednisolona se debe comenzar con una dosis de entre 30-60 $\mathrm{mg} /$ día, para con posterioridad ir disminuyendo progresivamente la dosis, hasta llegar a una dosis de mantenimiento de 5-10 mg/día que se puede mantener durante unos dos años ${ }^{2}$.

En el caso que nos ocupa, se inició tratamiento con metilprednisolona (Urbasón ${ }^{\circledR}$ ) 40 $\mathrm{mg} /$ día que debería de mantenerse durante el primer mes, para a continuación ir reduciendo la dosis de corticoide hasta llegar a una dosis de 8$10 \mathrm{mg} /$ día durante los meses que fuera menester. Al cabo de tres semanas y como consecuencia de su proceso base (pancreatitis reagudizada), el paciente presenta un fallo multiorgánico, que conlleva a un desenlace fatal.

\section{REFERENCIAS}

1. SERVER G, SERVER G Jr.: Fibrosis retroperitoneal. En: Tratado de Urología. Edited by Jiménez Cruz J.F. y Rioja Sanz L.A. Barcelona. JR Prous 1993; 1 (25): 417-429.

2. LEIVA O, DE LA FUENTE A, TEJIDO A, ZULUAGA A. CARBALLIDO JA, ÁLVAREZ-MON M.: Fibrosis retroperitoneal idiopática. En: Urología y Retroperitoneo. Editado por A. Zuluaga G. Tema Monográfico LXII Congreso Nacional de Urología. Junio 1997; Cap 6: 103-127.

3. BENEDICT MARTINA F, RETO NÜESCH, THOMAS C. GASSER. En: Fibrosis retroperitoneal y periarteritis crónica: una nueva hipótesis. European Urology 1994 marzo; 2: 117-120.

4. LEWIS JD, FAIGEL DO, MORRIS JB, SIEGELMAN ES, KOCHMAN ML.: In: Splenic vein thrombosis secondary to focal pancreatitis diagnosed by endoscopic ultrasonography. J Clin Gastroenterol 1998 jan; 26 (1): 54-56.

5. REYNAUD J, JARDEL P, CARRETIER M, COSTE G, TOUCHARD G, AUBERT J, BARBIER J. In: Pancreatitis: a little-known cause of ureteral obstruction. Ann Urol (Paris) 1984 sep; 18 (5): 313-317.

6. RUCKERT K, SCHWARZKOPF W, RUDIGIER J. In: Retroperitoneal fibrosis and pancreatitis. Leber Magen Darm 1978 feb; 8 (1): 12-16.

7. TURO ANTONA J, TORRES LEÓN J, PEIRO MARTÍNEZ F, CABRERA CABRERA JA. En: Uropatía obstructiva por fibrosis retroperitoneal secundaria a aneurisma de aorta abdominal. Actas Urol Esp 2000; 24 (9): 764-766.

Dr. M.A. García García

Servicio de Urología

Hospital Clínico de Salamanca

Po de San Vicente, 182

37007 Salamanca

(Trabajo recibido el 11 marzo de 2003) 